\title{
Preventive effect of olanzapine on trimethyltin neurotoxicity in mice: Evaluation of hippocampal neuronal loss, microglial activation, and cognitive dysfunction
}

\author{
Yusuke Onaka*, Shota Wada, Taro Yamaguchi, Masanori Yoneyama and Kiyokazu Ogita \\ Laboratory of Pharmacology, Faculty of Pharmaceutical Sciences, Setsunan University, Hirakata, Osaka 573-0101, Japan
}

\begin{abstract}
The organotin trimethyltin (TMT) induces neurodegeneration in the hippocampal dentate gyrus and psychiatric changes in mice such as cognitive dysfunction. We previously established a TMT-treated mouse model of cognitive dysfunction with hippocampal neurodegeneration. Olanzapine is an antipsychotic drug that has neuroprotective effects and improves cognitive dysfunction observed in schizophrenia. The present study aimed to elucidate the effects of olanzapine on cognitive dysfunction and hippocampal neurodegeneration in TMT-treated mice. Our results indicated that olanzapine effectively prevented TMT-induced neurotoxicity and the subsequent activation of microglia in the hippocampus, thus protecting hippocampal neurons from damage and preserving cognitive function. Minocycline (an inhibitor of microglial activation) also prevented TMT-induced neurotoxicity and demonstrated a synergistic effect with olanzapine. Collectively, our findings indicate that olanzapine may prevent TMT-related neurotoxicity, including hippocampal neurodegeneration and cognitive dysfunction.
\end{abstract}

\section{Introduction}

Hippocampal neuronal loss is a well-established pathology that causes cognitive dysfunction. Cognitive dysfunction due to progressive neurodegeneration occurs in a wide range of neurodegenerative disorders, including Alzheimer's disease, cerebral ischemic insults, and Parkinson's disease [1,2]. In addition to neurodegenerative diseases, the severity of symptoms in schizophrenia is correlated with a decrease in brain volume $[3,4]$.

Many reports have demonstrated that trimethyltin (TMT) induces neurodegeneration and the selective activation of glial cells in the hippocampal dentate gyrus of mice [5,6]. Immediately after TMTinduced neurodegeneration in the dentate gyrus, neurogenesis becomes markedly enhanced [7]. Accordingly, these features of TMT have become useful in the research on hippocampal neurodegeneration and neuroregeneration [8]. In addition, TMT induces abnormal behaviors, including cognitive dysfunction and depressive-like behaviors [7,9]. Previous studies have shown that TMT-treated mice are useful for analyzing psychiatric changes involved in neurodegeneration or neuroregeneration in the hippocampal dentate gyrus.

Olanzapine, a multi-acting, receptor-targeted antipsychotic, has been shown to improve not only the positive and negative symptoms of schizophrenia but also cognitive dysfunction in both schizophrenia and Alzheimer's disease [10]. In addition, previous studies have demonstrated that olanzapine is capable of preventing hydrogen peroxide, $\beta$ amyloid, and $\mathrm{N}$-methyl-4-phenylpyridinium ions from damaging PC12 cells [11-13]. Based on these findings, we hypothesized that olanzapine prevents TMT-induced neurotoxicity, including hippocampal neuronal degeneration and cognitive dysfunction. Therefore, the present study aimed to elucidate whether olanzapine improves TMT-induced neurodegeneration and cognitive dysfunction.

\section{Materials and methods}

\section{Animals}

The animal protocols used in this study met the guidelines of The Japanese Society for Pharmacology and were approved by the Committee for Ethical Use of Experimental Animals at Setsunan University. All efforts were made to minimize animal suffering, reduce the number of animals used, and utilize alternatives to in vivo techniques. Sevenweek-old male Std-ddY mice were housed 4-5/cage $(24 \times 17 \times 12 \mathrm{~cm} 3)$ in a temperature-controlled room and were maintained on a 12-h lightdark cycle (lights on from 7 AM to 7 PM). They were given free access to food and water for at least 4 days before use.

\section{Reagents}

Trimethyltin chloride (93-5084, Stream Chemicals, Inc. Newburyport, MA, USA) was dissolved in phosphate-buffered saline (PBS, pH 7.4). Olanzapine (156-03073, Wako Pure Chemical Industries, Ltd., Osaka, Japan) was dissolved in saline ( $\mathrm{pH}$ 6.5). Chloral hydrate (Nacalai Tesque, Inc. Kyoto, Japan) and minocycline hydrochloride (M9511, Sigma-Aldrich, St. Louis, Missouri, USA) were dissolved in saline. All drugs were intraperitoneally administered at a volume of 10 $\mathrm{mL} / \mathrm{kg}$. All other chemicals were of the highest purity commercially available.

*Correspondence to: Yusuke Onaka PhD, Laboratory of Pharmacology, Faculty of Pharmaceutical Sciences, Setsunan University, Hirakata, Osaka 573-0101, Japan, E-mail: yusuke.onaka@pharm.setsunan.ac.jp

Key words: cognitive dysfunction, microglia, neurodegeneration, olanzapine, trimethyltin

Received: August 16, 2018; Accepted: August 28, 2018; Published: August 30, 2018 


\section{Assessment of cognitive function}

Cognitive function was assessed by the novel object recognition test as previously described [14]. Briefly, each mouse was individually habituated to a Plexiglas box $(25.5 \times 30 \times 40 \mathrm{~cm})$, the floor of which was covered in sawdust. During habituation, mice were allowed to explore for $10 \mathrm{~min}$ in the absence of objects for 3 consecutive days. On day 4 , two objects were symmetrically placed on the floor of the box, and mice were allowed to explore the box for $10 \mathrm{~min}$ as part of the training session. The two objects were similar in size but different in shape and color. At $24 \mathrm{~h}$ after the training session, each animal was returned to the same box, in which one of the objects was replaced with a novel object. Mice were then allowed to explore the objects for $10 \mathrm{~min}$ as part of the retention session. This exploration behavior was calculated as the time spent sniffing each object. The preference index was calculated as the ratio of time spent exploring the novel object in the retention session to the total time spent exploring both objects.

\section{Histological assessments}

Mice were deeply anesthetized with chloral hydrate $(500 \mathrm{mg} /$ $\mathrm{kg})$, followed by transcardial perfusion with saline and $4 \%(\mathrm{w} / \mathrm{v})$ paraformaldehyde in PBS as fixation. The whole brains were dissected and post-fixed in the same fixative solution overnight at $4^{\circ} \mathrm{C}$. Post-fixed brains were embedded in paraffin and cut with a microtome into three sagittal sections of $5-\mu \mathrm{m}$ thickness at $240-\mu \mathrm{m}$ intervals ranging from 0.7 $\mathrm{mm}$ to $1.2 \mathrm{~mm}$ to lateral. The brain sections were deparaffinized with xylene, rehydrated by immersion in graded decreasing concentrations of ethanol from $100 \%(\mathrm{vol} / \mathrm{vol})$ to $50 \%(\mathrm{vol} / \mathrm{vol})$, and washed with deionized water.

To detect damaged neurons, Fluoro-Jade B (FJB) staining was performed as previously described [15]. Briefly, sections were blocked with $0.06 \%(\mathrm{w} / \mathrm{v}) \mathrm{KMnO} 4$ solution. After washing with deionized water, sections were incubated for 20 min with $0.0002 \%$ FJB (G310, Millipore Ltd., Tokyo, Japan) solution dissolved in $0.1 \%(\mathrm{v} / \mathrm{v})$ acetic acid. After washing with deionized water, sections were dried at $60^{\circ} \mathrm{C}$ and dehydrated using $100 \%$ xylene for over $1 \mathrm{~min}$.

Iba-1 immunostaining was performed as previously described [16]. Briefly, rehydrated sections were heated for $10 \mathrm{~min}$ in a microwave oven in $10 \mathrm{mM}$ sodium citrate buffer ( $\mathrm{pH}$ 7.0) for antigen activation. After washing with Tris-buffered saline containing $0.05 \%(\mathrm{w} / \mathrm{v})$ Tween 20 (TBST), sections were blocked with $5 \%(\mathrm{w} / \mathrm{v})$ normal goat serum for 1 $\mathrm{h}$ at room temperature, followed by incubation with rabbit anti-mouse Iba-1 antibody (1:1000 dilution; 019-19741, Wako Pure Chemical Industries) at $4^{\circ} \mathrm{C}$ overnight. After washing with TBST, sections were incubated with Alexa Fluor 594 Goat anti-rabbit IgG $(\mathrm{H}+\mathrm{L})$ antibody (1:1000; R37117, Thermo Fisher Scientific Inc. Waltham, MA, USA) at room temperature for $2 \mathrm{~h}$.

Stained sections were viewed with a BX41 microscope (Olympus, Tokyo, Japan) equipped with a DS-Ril camera (Nikon, Tokyo, Japan). For FJB staining, the number of highly labeled cells was counted by microscopic observation. For Iba-1 staining, cells were classified as either resting or reactive microglia according to established morphological criteria [16]. Cells with less than 2 cell processes or 3-5 short branches were defined as resting microglia. Reactive microglia were identified according to particular histological characteristics, which included more than 5 long cell processes, a large soma with retracted and thicker processes, an amoeboid cell body, and numerous short processes. Three sagittal sections prepared from the brain of each animal were used for immunostaining and counting positive cells. The number of positive cells was represented as the average of three sections per animal.

\section{Statistical analysis}

Statistical analysis was performed using JMP Pro (SAS Institute Japan Ltd., Tokyo, Japan). Significant differences were determined using one-way or two-way analysis of variance followed by Tukey's honesty significant difference tests. $\mathrm{P}<0.05$ was considered statistically significant.

\section{Results}

\section{Olanzapine prevented TMT-induced cognitive dysfunction}

We have previously shown that acute injection with TMT causes substantial neuronal loss in the dentate granule cell layer on day 2 posttreatment as well as cognitive dysfunction in mice [7]. To evaluate the effect of olanzapine on TMT-induced cognitive dysfunction, the novel object recognition test was performed in the present study (Figure 1). TMT significantly decreased the exploratory preference of the mice in the retention session, without affecting that in the training session. Olanzapine completely abolished this decrease in exploratory preference in TMT-treated animals but did not affect control animals treated with PBS. However, olanzapine failed to affect the exploratory preference in the training session for animals treated with TMT or PBS. These data suggest that olanzapine prevented TMT-induced cognitive dysfunction.

\section{Olanzapine prevented TMT from damaging neuronal cells and activating microglia in the dentate gyrus}

To evaluate the effect of olanzapine on TMT-induced neurodegeneration in the hippocampal dentate gyrus, FJB staining (Figure 2A), a well-established method for staining degenerating neurons [17], was performed. TMT induced substantial generation of FJB-positive cells selective to the granule cell layer of the hippocampal dentate gyrus (Figure 2A \& 2B). Interestingly, olanzapine significantly decreased the number of FJB-positive cells in the dentate gyrus of

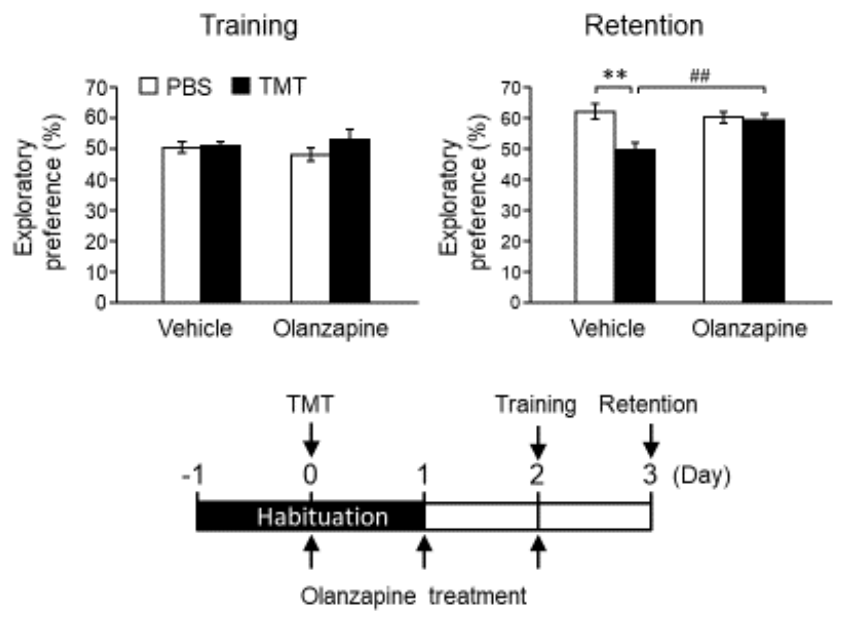

Figure 1. Effect of olanzapine on TMT-induced cognitive dysfunction Mice were intraperitoneally injected with either PBS or TMT $(2.6 \mathrm{mg} / \mathrm{kg})$. Olanzapine ( 0.6 $\mathrm{mg} / \mathrm{kg}$ ) or vehicle (saline) was intraperitoneally injected into the mice at $30 \mathrm{~min}, 24 \mathrm{~h}$, and $48 \mathrm{~h}$ after TMT injection (lower panel). The left graph denotes the exploratory preference for one object in the training session of the novel object recognition test. The right graph denotes the exploratory preference for the novel object in the retention session of the novel object recognition test. Data are expressed mean $\pm \operatorname{SEM}(\mathrm{n}=14) . * * P<0.01$, significantly different between each value obtained for animals treated with PBS or TMT. ${ }^{\#} P<0.01$, significantly different between each value obtained for animals treated with vehicle or olanzapine. 

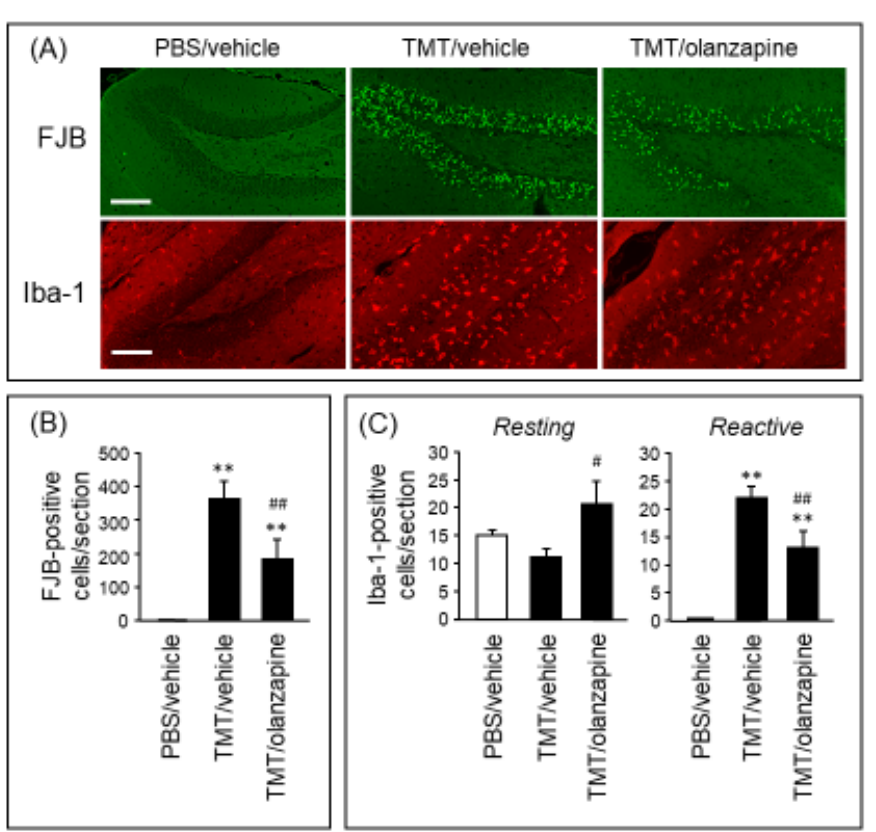

Figure 2. Effect of olanzapine on TMT-induced neurodegeneration and microglial activation

Mice were intraperitoneally injected with either PBS or TMT $(2.6 \mathrm{mg} / \mathrm{kg})$. Olanzapine $(0.6$ $\mathrm{mg} / \mathrm{kg}$ ) or vehicle (saline) was intraperitoneally injected into the mice at $30 \mathrm{~min}$ and $24 \mathrm{~h}$ after TMT injection. The mice were decapitated at $24 \mathrm{~h}$ after the final injection. (A) Typical fluorescence micrographs of FJB staining and Iba-1 immunostaining in the hippocampal dentate gyrus of animals treated with PBS or vehicle (PBS/vehicle), TMT or vehicle (TMT/ vehicle), and TMT or olanzapine (TMT/olanzapine). Scale bar $=100 \mu \mathrm{m}$. (B) The number of FJB-positive cells per section of the dentate gyrus slice. Data are expressed as mean \pm SEM $(n=8)$. (C) The number of Iba-1-positive cells (resting and reactive forms) per section of the dentate gyrus slice. Data are expressed as mean \pm SEM. (PBS/vehicle, $n=7$; TMT/ vehicle, $\mathrm{n}=7$; TMT/olanzapine, $\mathrm{n}=6$ ). $* * P<0.01$, significantly different from the value obtained in PBS/vehicle. ${ }^{\#} P<0.05,{ }^{\# P} P<0.01$, significantly different between TMT/vehicle and TMT/olanzapine.

TMT-treated animals. These data suggest that olanzapine prevented TMT from damaging neuronal cells in the dentate gyrus.

It is well-known that TMT induces microglial activation accompanied by neurodegeneration $[18,19]$ and that activated microglia play a role in neurodegeneration [20]. Therefore, Iba-1 immunostaining was performed to evaluate microglia and the effect of olanzapine on TMT-induced microglial activation (Figure 2A). Systemic treatment with TMT generated a substantial increase in Iba1-positive cells selective to the granule cell layer, molecular layer, and hilus of the hippocampal dentate gyrus. In addition, TMT substantially increased the number of Iba-1-positive cells characterized as reactive microglia (reactive form) but did not significantly affect the number of those characterized as resting microglia in the dentate gyrus (resting form, Figure 2A \& 2C). In TMT-treated animals, olanzapine decreased the number of reactive Iba-1-positive cells and conversely, increased the number of resting Iba-1-positive cells. These data suggest that olanzapine suppressed TMT-induced microglial activation in the dentate gyrus.

\section{Minocycline prevented TMT from damaging neuronal cells and activating microglia in the dentate gyrus}

To elucidate the involvement of reactive microglia in TMT-induced neurodegeneration, the effect of minocycline, an inhibitor of microglial activation, on TMT-induced neurodegeneration and microglial activation (Figure 3) was assessed. As expected, minocycline decreased the number of reactive Iba-1-positive cells in TMT-treated animals (Figure 3A \& 3C). In addition, minocycline significantly decreased the number of FJB-positive cells generated by TMT (Figure 3A \& 3B). These data suggest that inhibition of microglial activation served to suppress TMT-induced neurodegeneration in the dentate gyrus.

\section{Effect of co-administration of olanzapine and minocycline on TMT-induced neurodegeneration in the dentate gyrus}

The effect of co-administration of olanzapine and minocycline on TMT-induced neurodegeneration was assessed (Figure 4). In TMTgenerated FJB-positive cells, the preventive effect of co-administration of olanzapine and minocycline was stronger than that of administration of either compound alone. However, TMT-induced activation of microglia was inhibited by co-administration of olanzapine and minocycline. This inhibitory effect of the co-administration was comparable with that of olanzapine or minocycline alone (data not shown).

\section{Discussion}

It is well established that olanzapine has neuroprotective effects in vitro and improves cognitive dysfunction in vivo. However, few studies have revealed the role of olanzapine in neurodegenerationrelated cognitive dysfunction. The present study demonstrated for the first time that olanzapine prevents TMT from causing cognitive dysfunction, neurodegeneration, and microglial activation. In addition,
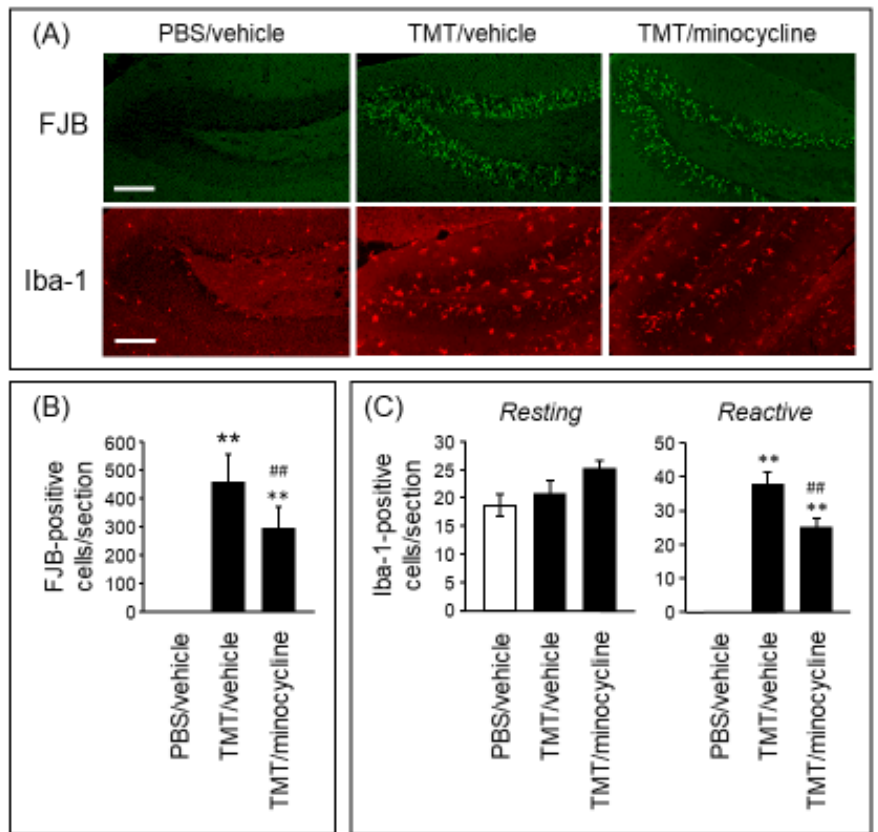

Figure 3. Effect of minocycline on TMT-induced neurodegeneration and microglial activation

Mice were intraperitoneally injected with either PBS or TMT ( $2.6 \mathrm{mg} / \mathrm{kg})$. Minocycline (50 $\mathrm{mg} / \mathrm{kg}$ ) or vehicle (saline) was intraperitoneally injected into the mice at $30 \mathrm{~min}$ and $24 \mathrm{~h}$ after TMT injection. The mice were decapitated at $24 \mathrm{~h}$ after the final injection. (A) Typical fluorescence micrographs of FJB staining and Iba-1 immunostaining in the hippocampal dentate gyrus of animals treated with PBS or vehicle (PBS/vehicle), TMT or vehicle (TMT/ vehicle), and TMT or minocycline (TMT/minocycline). Scale bar $=100 \mu \mathrm{m}$. (B) The number of FJB-positive cells per section of the dentate gyrus slice. Data are expressed as mean \pm SEM $(n=8)$. (C) The number of Iba-1-positive cells (resting and reactive forms) per section of the dentate gyrus slice. Data are expressed as mean \pm SEM (PBS/vehicle, $n$ $=7 ; \mathrm{TMT} /$ vehicle, $\mathrm{n}=7 ; \mathrm{TMT} /$ minocycline, $\mathrm{n}=6) . * * P<0.01$, significantly different from the value obtained in PBS/vehicle. ${ }^{\#} P<0.01$, significantly different between TMT/vehicle and TMT/minocycline. 

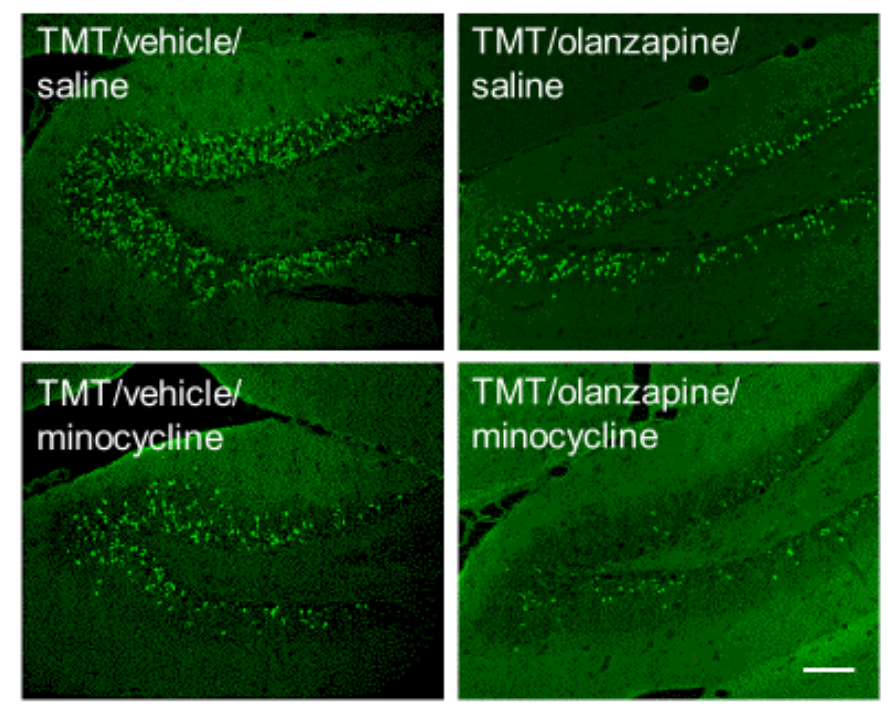

Figure 4. Additive effect of olanzapine and minocycline on TMT-induced neurodegeneration Mice were intraperitoneally injected with TMT $(2.6 \mathrm{mg} / \mathrm{kg})$. Olanzapine $(0.6 \mathrm{mg} / \mathrm{kg})$, minocycline $(50 \mathrm{mg} / \mathrm{kg}$ ), or vehicle (saline) was intraperitoneally injected into the mice at $30 \mathrm{~min}$ and $24 \mathrm{~h}$ after TMT injection. The mice were decapitated at $24 \mathrm{~h}$ after the final injection. Typical fluorescence micrographs reveal FJB staining in the hippocampal dentate gyrus of animals treated with drugs as indicated. Experiments were conducted at least three times, with similar results obtained under the same experimental conditions. Scale bar = $100 \mu \mathrm{m}$

we found that microglial activation is, at least partly, involved in TMTinduced neurodegeneration. The finding that co-administration of olanzapine and minocycline had a stronger neuroprotective effect than that of each drug alone allowed us to hypothesize that olanzapine elicits a neuroprotective effect against TMT neurotoxicity likely through the suppressed activation of microglia, along with other unknown mechanisms. A previous in vivo study in rats has provided evidence for the neuroprotective effect of olanzapine by demonstrating that olanzapine improves neuronal death and cognitive dysfunction induced by the intra-hippocampal administration of okadaic acid [20]. Our current findings of the neuroprotective effect of olanzapine against TMT-induced neurotoxicity, in addition to those of okadaic acid, support the proposition that olanzapine has universal mechanisms for neuroprotection. Elucidation of the unknown mechanisms underlying this neuroprotection may contribute to new treatments for neurodegenerative disorders.

Previous reports have demonstrated that TMT causes hippocampal neurodegeneration and cognitive dysfunction [7,21]. Further evidence for the involvement of hippocampal damage in cognitive dysfunction has been provided by various reports on colchicine-induced lesions of the hippocampal dentate gyrus [22] and amyloid $\beta$-induced neuronal death in the dentate gyrus [23]. Thus, cognitive dysfunction induced by TMT is attributed to neurodegeneration in the dentate gyrus. This proposition is supported by our previous studies reporting that TMT induces temporary cognitive dysfunction, which is abolished by regeneration after loss of granule cells in the dentate gyrus [7,9].

Because our findings indicate that minocycline suppressed TMTinduced neurodegeneration, it appears that microglial activation is involved in TMT-induced neurodegeneration. Accumulating evidence suggests that the pro-inflammatory activation of microglia is involved in the pathology of various neurodegenerative disorders such as Parkinson's disease, Alzheimer's disease, multiple sclerosis, and AIDS dementia [24-26]. Furthermore, minocycline has been shown to inhibit neurodegeneration induced by 1-methyl-4-phenyl- 1,2,3,6-tetrahydropyridine and amyloid $\beta$ in in vivo studies $[27,28]$. Our results that minocycline prevented TMT neurotoxicity strongly support the notion that TMT neurotoxicity is due to, at least partly, the pro-inflammatory activation of microglia. Importantly, the present in vivo study also demonstrated that olanzapine prevented TMT-induced microglia activation and neurodegeneration. Based on the findings of previous in vitro studies demonstrating that olanzapine inhibited microglial proton currents or the release of nitric oxide in microglial cell lines $[29,30]$, we proposed that olanzapine directly contributes to the inhibition of microglial activation. Therefore, olanzapine inhibits TMT-induced neurodegeneration through the direct inhibition of microglia activation.

Several studies have reported on the effects of olanzapine on cell damage through different mechanisms in numerous cells. In PC12 cells, olanzapine enhances superoxide dismutase activity, thus protecting cells from death induced by hydrogen peroxide or N-methyl-4phenylpyridinium ions $[11,13]$. In addition, olanzapine regulates the translocation and expression of the pro- and anti-apoptotic proteins Bax and Bcl-XL, respectively, in PC12 cells [31]. Furthermore, an in vivo study suggested that chronic administration of olanzapine upregulates the expression of $\mathrm{Bcl}-2$ in the hippocampus [32]. These reports support the proposition that olanzapine has both antioxidant and anti-apoptotic effects, which contribute to its neuroprotective effects against TMTinduced neurodegeneration.

\section{Conclusion}

In conclusion, our current study provides evidence suggesting that olanzapine improves cognitive dysfunction accompanied by neurodegeneration. Moreover, olanzapine appeared to prevent neurodegeneration through the inhibition of microglial activation, indicating that it may be useful as a therapeutic drug for acute neurodegenerative disorders.

\section{Conflict of interest}

The authors declare no conflict of interest.

\section{References}

1. Pena-Altamira E, Petralla S, Massenzio F, Virgili M, Bolognesi ML, et al. (2017) Nutritional and Pharmacological Strategies to Regulate Microglial Polarization in Cognitive Aging and Alzheimer's Disease. Front Aging Neurosci 9: 175. [Crossref]

2. Collins LM, Williams-Gray CH (2016) The Genetic Basis of Cognitive Impairment and Dementia in Parkinson's Disease. Front Psychiatry 7: 89. [Crossref]

3. Hulshoff Pol HE, Kahn RS (2008) What happens after the first episode? A review of progressive brain changes in chronically ill patients with schizophrenia. Schizophr Bull 34: 354-366. [Crossref]

4. Andreasen NC, Nopoulos P, Magnotta V, Pierson R, Ziebell S, et al. (2011) Progressive Brain Change in Schizophrenia: A Prospective Longitudinal Study of First-Episode Schizophrenia. Biol Psychiatry 70: 672-679. [Crossref]

5. Bruccoleri A, Brown H, Harry GJ (1998) Cellular localization and temporal elevation of tumor necrosis factor-alpha, interleukin-1 alpha, and transforming growth factorbeta 1 mRNA in hippocampal injury response induced by trimethyltin. $J$ Neurochem 71: 1577-1587. [Crossref]

6. Reuhl KR, Smallridge EA, Chang LW, Mackenzie BA (1983) Developmental effect of trimethyltin intoxication in the neonatal mouse. I. Light microscopic studies. Neurotoxicology 4: 19-28. [Crossref]

7. Ogita K, Nishiyama N, Sugiyama C, Higuchi K, Yoneyama M, et al. (2005) Regeneration of granule neurons after lesioning of hippocampal dentate gyrus: evaluation using adult mice treated with trimethyltin chloride as a model. $J$ Neurosci Res 82: 609-621. [Crossref]

8. Lee S, Yang M, Kim J, Kang S, Kim J, et al. (2016) Trimethyltin-induced hippocampal neurodegeneration: A mechanism-based review. Brain Res Bull 125: 187-199. [Crossref] 
9. Yoneyama M, Shiba T, Hasebe S, Umeda K, Yamaguchi T, et al. (2014) Lithium promotes neuronal repair and ameliorates depression-like behavior following trimethyltin-induced neuronal loss in the dentate gyrus. PLoS One 9: e87953. [Crossref]

10. Kirkpatrick B, Buchanan RW, Ross DE, Carpenter WT Jr (2001) A separate disease within the syndrome of schizophrenia. Arch Gen Psychiatry 58: 165-171. [Crossref]

11. Wei Z, Bai O, Richardson JS, Mousseau DD, Li XM (2003) Olanzapine protects PC12 cells from oxidative stress induced by hydrogen peroxide. J Neurosci Res 73: 364-368. [Crossref]

12. Wang H, Xu H, Dyck LE, Li XM (2005) Olanzapine and quetiapine protect PC12 cells from beta-amyloid peptide(25-35)-induced oxidative stress and the ensuing apoptosis. J Neurosci Res 81: 572-580. [Crossref]

13. Park SW, Lee CH, Lee JG, Kim LW, Shin BS, et al. (2011) Protective effects of atypical antipsychotic drugs against MPP(+)-induced oxidative stress in PC12 cells. Neurosci Res 69: 283-290. [Crossref]

14. Onaka Y, Shintani N, Nakazawa T, Kanoh T, Ago Y, et al. (2016) Prostaglandin D2 signaling mediated by the CRTH2 receptor is involved in MK-801-induced cognitive dysfunction. Behav Brain Res 314: 77-86. [Crossref]

15. Tanaka M, Yoneyama M, Shiba T, Yamaguchi T, Ogita K (2016) Protease-activated receptor-1 negatively regulates proliferation of neural stem/progenitor cells derived from the hippocampal dentate gyrus of the adult mouse. J Pharmacol Sci 131: 162171. [Crossref]

16. Yoneyama M, Hasebe S, Kawamoto N, Shiba T, Yamaguchi T, et al. (2014) Beneficia in vivo effect of aripiprazole on neuronal regeneration following neuronal loss in the dentate gyrus: evaluation using a mouse model of trimethyltin-induced neuronal loss/ self-repair in the dentate gyrus. J Pharmacol Sci 124: 99-111. [Crossref]

17. Schmued LC, Albertson C, Slikker W Jr (1997) Fluoro-Jade: a novel fluorochrome for the sensitive and reliable histochemical localization of neuronal degeneration. Brain Res 751: 37-46. [Crossref]

18. Kim DJ, Kim YS. Trimethyltin-Induced Microglial Activation via NADPH Oxidase and MAPKs Pathway in BV-2 Microglial Cells. Mediators of inflammation. 2015;2015:729509. [Crossref]

19. Pompili E, Fabrizi C, Nori SL, Panetta B, Geloso MC, et al. (2011) Protease-activated receptor-1 expression in rat microglia after trimethyltin treatment. $J$ Histochem Cytochem 59: 302-311. [Crossref]

20. He J, Yang Y, Xu H, Zhang X, Li XM (2005) Olanzapine attenuates the okadaic acid-induced spatial memory impairment and hippocampal cell death in rats. Neuropsychopharmacology 30: 1511-1520. [Crossref]
21. Ogita K, Nitta Y, Watanabe M, Nakatani Y, Nishiyama N, et al. (2004) In vivo activation of c-Jun N-terminal kinase signaling cascade prior to granule cell death induced by trimethyltin in the dentate gyrus of mice. Neuropharmacology 47: 619-630. [Crossref]

22. Xavier GF, Oliveira-Filho FJ, Santos AM (1999) Dentate gyrus-selective colchicine lesion and disruption of performance in spatial tasks: difficulties in "place strategy" because of a lack of flexibility in the use of environmental cues? Hippocampus 9: 668681. [Crossref]

23. Moon M, Choi JG, Nam DW, Hong HS, Choi YJ, et al. (2011) Ghrelin ameliorates cognitive dysfunction and neurodegeneration in intrahippocampal amyloid-beta1-42 oligomer-injected mice. J Alzheimers Dis 23: 147-159. [Crossref]

24. Gao HM, Liu B, Zhang W, Hong JS (2003) Novel anti-inflammatory therapy for Parkinson's disease. Trends Pharmacol Sci 24: 395-401. [Crossref]

25. McGeer PL, McGeer EG (1995) The inflammatory response system of brain implications for therapy of Alzheimer and other neurodegenerative diseases. Brain Res Brain Res Rev 21: 195-218. [Crossref]

26. Minagar A, Shapshak P, Fujimura R, Ownby R, Heyes M, et al. (2002) The role of macrophage/microglia and astrocytes in the pathogenesis of three neurologic disorders: HIV-associated dementia, Alzheimer disease, and multiple sclerosis. Journal of the neurological sciences. 202: 13-23. [Crossref]

27. Wu DC, Jackson-Lewis V, Vila M, Tieu K, Teismann P, et al. (2002) Blockade of microglial activation is neuroprotective in the 1-methyl-4-phenyl-1,2,3,6tetrahydropyridine mouse model of Parkinson disease. J Neurosci 22: 1763-1771. [Crossref]

28. Ryu JK, Franciosi S, Sattayaprasert P, Kim SU, McLarnon JG (2004) Minocycline inhibits neuronal death and glial activation induced by beta-amyloid peptide in rat hippocampus. Glia 48: 85-90. [Crossref]

29. Shin H, Kim J, Song JH (2015) Clozapine and olanzapine inhibit proton currents in BV2 microglial cells. Eur J Pharmacol 755: 74-79. [Crossref]

30. Hou Y, Wu CF, Yang JY, He X, Bi XL, et al. (2006) Effects of clozapine, olanzapine and haloperidol on nitric oxide production by lipopolysaccharide-activated N9 cells. Prog Neuropsychopharmacol Biol Psychiatry 30: 1523-1528. [Crossref]

31. Wei Z, Mousseau DD, Richardson JS, Dyck LE, Li XM (2003) Atypical antipsychotic attenuate neurotoxicity of beta-amyloid(25-35) by modulating Bax and $\mathrm{Bcl}-\mathrm{X}(1 / \mathrm{s})$ expression and localization. J Neurosci Res 74: 942-947. [Crossref]

32. Bai O, Zhang H, Li XM (2004) Antipsychotic drugs clozapine and olanzapine upregulate bcl-2 mRNA and protein in rat frontal cortex and hippocampus. Brain Res 1010: 81-86. [Crossref]

Copyright: (C2018 Onaka Y. This is an open-access article distributed under the terms of the Creative Commons Attribution License, which permits unrestricted use, distribution, and reproduction in any medium, provided the original author and source are credited. 